ORIGINAL ARTICLE

PRACA ORYGINALNA

\title{
BONE REMODELING FEATURES IN ELDERLY AND SENILE PATIENTS WITH THE PROXIMAL FEMUR FRACTURES AFTER HIP REPLACEMENT
}

DOI: 10.36740/WLek202002110

\author{
Oleksandr M. Khvysyuk, Volodymyr O. Babalian, Serge B. Pavlov, Galina B. Pavlova \\ KHARKIV MEDICAL ACADEMY OF POSTGRADUATE EDUCATION, KHARKIV, UKRAINE
}

\begin{abstract}
The aim of this study is to identify the dependence of the result of surgical treatment of patients of elderly and senile age with fractures of the proximal femur on the characteristics of the response cytokine-mediated regulatory response to trauma and surgery.

Materials and methods: In 74 patients after hip arthroplasty, serum levels of bone metabolism markers were determined using enzyme-linked immunosorbent assay. Patients were divided into 2 groups depending on the results of treatment.

Results: It was found that compared with group 2 (treatment outcome is worse) in group 1 (treatment outcome is better) there was a greater number of correlations. In group 1, correlations were found between OPG and RANKL $(r=0.88 ; p=0.000), 0 P G$ and OPG/RANKL $(r=0.44 ; p=0.006)$, TGF- $\beta 1$ and OPG/RANKL $(r=0.66 ; p=0.000), I L-6$ and OPG $(r=0.67 ; p=0.000)$, IL-6 and RANKL $(r=0.53 ; p=0.001)$, IL-6 and OPG/RANKL $(r=0.39 ; p=0.016)$. In group 2 , only between OPG and OPG/RANKL $(r=0.72 ; p=$ $0.000)$, RANKL and OPG/RANKL $(r=-0.53 ; p=0.0007)$. In patients of group 2, there was a decrease in the level of OPG relative to the control and a less significant increase in TGF- $\beta 1$ and IL-6 relative to group 1.

Conclusion: The prognosis of the results of treatment of patients with proximal femur fractures is largely determined by the nature of the adaptive response to injury and the implant, the synchronism of the mechanism of stress remodeling of the bone. A less favorable prognosis after arthroplasty is associated with exacerbation of the initial metabolic disorders in the bone tissue due to severe cytokine-mediated dysfunction of the regulatory pathways.
\end{abstract}

KEY WORDS: hip arthroplasty, bone remodeling, RANK ligand, osteoprotegerin, transforming growth factor- $\beta 1$, interleukin- 6

Wiad Lek. 2020;73(2):259-265

\section{INTRODUCTION}

Development of systemic osteoporosis worldwide provokes an increasing number of proximal femur fractures. Osteosynthesis and endoprosthetics are generally accepted methods of such fractures surgical treatment. However, long-term results after plate osteosynthesis are often unsatisfactory (up to $87.5 \%$ of cases) [1]. The method of hip arthroplasty is more effective and contributes to the achievement of functional recovery in the early stages.

It is known that the risk of unsatisfactory results of arthroplasty increases with the age of the patient. A feature of patients of older age groups is the combination of 2 or more diseases that can be mutually aggravating. However, it remains unclear why with similar clinical conditions; some patients suffer trauma and surgery, while others do not, in some implants fail, and in others remain stable. Evaluation and prediction of treatment outcomes is a difficult task, since the decision must be based on a comprehensive assessment of many factors, including from the point of view of a system analysis of the state of adaptation reserves of the body.

The reaction to damage is determined by the state of the connective tissue, the reserves of adaptation and the ability to maintain homeostasis. This is the basis of the pro- cesses: inflammation and regeneration, the development of a stereotypical reaction of the body to damage. These processes are basic for acute surgical pathology; determine the current and long-term prognosis [2].

The clinical outcome of the disease is associated precisely with the reaction of the connective tissue, as a physiological system, as one of the most important components of the systemic inflammatory response syndrome [3]. For elderly patients, this becomes critically important, not only because we are talking about bone damage in the background of osteoporosis, but primarily because the physiological reserves of the body are significantly reduced.

The nature, depth, duration of the inflammatory process and the body's immune response regulate cytokines. The change in their level is a reflection of the functioning of the regulatory systems of the body, has an impact on the main parts of the pathological process [4]. The fracture healing process can be considered as a form of tissue regeneration. Regeneration processes are a complex interweaving of general effects at the system level and local changes in tissue metabolism, including changes at the molecular level. The result of the adaptive multifactor reaction of bone tissue to the implant is stress remodeling, which largely depends on the state of regulatory mechanisms that determine the 
enhancement of bone resorption and the failure of repair processes. Consequently, to assess adaptation reserves and highlight informative prognostic criteria, it is important to assess the state of regulatory pathways, such as RANKRANKL-OPG and others, which include cytokines.

\section{THE AIM}

The aim of the work is to identify the dependence of the result of surgical treatment of elderly and senile patients with fractures of the proximal femur on the characteristics of the response cytokine-mediated regulatory response to trauma and surgical intervention.

\section{MATERIALS AND METHODS}

74 patients with proximal femur fractures elderly and senile age were examined. The average age of patients was $77.6 \pm 4.9$ years, among them 24 men and 50 women. All patients were treated and were operated on under conditions of the trauma department of the Kharkiv City Clinical Multidisciplinary Hospital No. 17 from 2008 to 2017 inclusive. The research was carried out in accordance with the requirements of the Helsinki Declaration (2008, 2013 revision) with the approval of the local Bioethical Committee. Signed informed consent allowing use of their data in this study was obtained from all patients. The patients were thoroughly informed about the purposes and procedures of the study before they gave written consent.

Patients with type A fractures were examined according to the international classification of fractures of the proximal femur in joint-stock companies (extra-articular, trochanteric fractures). Primary fractures of 31A2 (61.5\%) were diagnosed (periarticular fragmental fractures of the acetabular zone), simple fractures of the acetabular zone $(31 \mathrm{~A} 1)-$ in $5.8 \%$ of cases, and inter-turn fractures (31A3) - in $23.1 \%$ of cases. $9.6 \%$ of cases were other types of fractures. All patients underwent cement bipolar hemiarthroplasty with augmentation, fixation with needles and/or cerclege. A bipolar stainless steel endoprosthesis (Narang, bipolar hip prosthesis; TIPSAN, STAINLESS STEE), bone cement from EVOCEM 1 40g, and Kirschner knitting needles $1.2 \mathrm{~mm}$ in diameter were used.

Almost all patients had cardiovascular system pathology: $90 \%$ had coronary heart disease, $87 \%$ had hypertension (mainly 2 degrees). Diseases of other organs and systems were less common. Among them, 25\% of patients had neurological disorders (consequences of strokes, dyscirculatory encephalopathy), $5 \%$ had diabetes, $9 \%$ had pathology of the gastrointestinal tract (erosive gastritis and pancreatitis), $10 \%$ had respiratory and urinary tract diseases.

Patients whose survival rate was more than 18 months were included. Treatment results were evaluated 12 months after surgery. A clinical evaluation of the results was carried out using the Harris Hip Score [7] rating scale to evaluate the results of treatment of hip fractures. Then Harris Hip Score answers were scored and the median was calculated. Depending on the number of points scored, all patients were divided into 2 groups: 1 group - 37 patients with a score higher than the median value (treatment results are better), 2 group - 37 patients with a score lower than the median value (treatment results are worse). The control group was 37 persons representative by age, sex and the presence of concomitant diseases.

The inclusion and exclusion criteria were formulated in such a way that the sample most closely corresponded to the general population of elderly and senile patients with fractures of the proximal femur. Therefore, patients were included in the study regardless of the type of concomitant pathology. Patients with infectious postoperative complications within 1 month after surgery, a history of co-infection (pneumonia, urinary tract infection), and a surgical procedure within 3 months before admission were excluded from the study.

The serum enzyme-linked immunosorbent assay determined the cytokine content: receptor activator of nuclear factor kappa-B ligand (RANKL) (Biomedica, Austria), osteoprotegerin (OPG) (Bioscience, Austria), transformed growth factor- $\beta 1$ (TGF- $\beta 1$ ) (DRG, Germany), interleukin-6 (IL-6) (Vector-Best, Russia). OPG/RANKL ratio was calculated. Studies were conducted on the 21st day after surgery. Statistical analysis was performed using the Statistica 6.0 package using the variance statistics methods ANOVA (Fisher LCD post-hoc test). Differences were considered statistically significant at a p-level $<0.05$.

\section{RESULTS}

It was established that the level of RANKL in patients of both groups had no significant differences with the control (table I). An increase level of RANKL in patients of group 2 relative to the control group can be regarded as differences at the level of the statistical trend $(p=0.07)$. The level of OPG compared with control was significantly reduced in patients of group 2. The ratio of OPG/RANKL compared with the control was lower in patients of groups 1 and 2 (2.6 and 3 times, respectively). Differences between the studied groups for all the above indicators were not identified.

The content of serum TGF- $\beta 1$ relative to the control increased in patients of both groups, most significantly in patients of group 1. Its level was significantly higher in patients of group 1 as compared with group 2. Its level was significantly higher in patients of group 1 as compared with group 2. In patients of group 2, the level of this cytokine was significantly lower than in group 1 .

In patients of group 1, there was a strong positive correlation between OPG and RANKL $(\mathrm{r}=0.88, \mathrm{p}=0.000)$ and $\mathrm{a}$ moderate strength positive relationship with a high level of statistical significance between the level of OPG and the ratio OPG/RANKL ( $\mathrm{r}=0.44, \mathrm{p}=0.006)$ (Fig. 1$)$.

In patients of group 2, a strong positive relationship of a high degree of significance between the OPG level and the OPG/RANKL ratio $(\mathrm{r}=0.72, \mathrm{p}=0.000)$ was revealed, as well as a negative relationship of the average power of a high degree of significance between the RANKL level and the OPG/RANKL ratio $(r=-0.53, p=0.0007)$. At the 
Table I. The cytokines content in the serum of patients depending on the results of treatment

\begin{tabular}{ccccc}
\hline Parameters & Control group & $\begin{array}{c}\mathbf{1} \text { group } \\
\mathbf{p} \text { - significance level of } \\
\text { differences with control }\end{array}$ & $\begin{array}{c}\mathbf{2} \text { group } \\
\mathbf{p}-\text { significance level of } \\
\text { differences with control }\end{array}$ & $\begin{array}{c}\text { Significance level of } \\
\text { differences between } \\
\text { groups } \mathbf{1} \text { and } \mathbf{2} \text { ( } \mathbf{p})\end{array}$ \\
\hline RANKL, pmol/l & $0.21 \pm 0.02$ & $\begin{array}{c}0.23 \pm 0.02 \\
\mathrm{p}=0.52\end{array}$ & $\begin{array}{c}0.26 \pm 0.02 \# \\
\mathrm{p}=0.07\end{array}$ & $\mathrm{p}=0.23$ \\
\hline $\mathrm{OPG}, \mathrm{pg} / \mathrm{ml}$ & $63.2 \pm 4.32$ & $\begin{array}{c}51.7 \pm 6.88 \\
\mathrm{p}=0.102\end{array}$ & $\begin{array}{c}45.0 \pm 2.68^{*} \\
\mathrm{p}=0.011\end{array}$ & $\mathrm{p}=0.344$ \\
\hline $\mathrm{OPG} / \mathrm{RANKL}$ & $574 \pm 116$ & $\begin{array}{c}224 \pm 15.0^{*} \\
\mathrm{p}=0.00043\end{array}$ & $\begin{array}{c}188 \pm 13.3^{*} \\
\mathrm{p}=0.00011\end{array}$ & $\mathrm{p}=0.708$ \\
\hline $\mathrm{TGF}-\beta 1, \mathrm{ng} / \mathrm{ml}$ & $11.9 \pm 0.35$ & $\begin{array}{c}15.5 \pm 0.55^{*} \\
\mathrm{p}=0.000\end{array}$ & $\begin{array}{c}13.4 \pm 0.46^{* *} \\
\mathrm{p}=0.026\end{array}$ & $\mathrm{p}=0.001$ \\
\hline $\mathrm{IL}-6, \mathrm{pg} / \mathrm{ml}$ & $3.72 \pm 0.232$ & $\begin{array}{c}13.2 \pm 1,27^{*} \\
\mathrm{p}=0,000\end{array}$ & $\begin{array}{c}6,17 \pm 0,75^{* *} \\
\mathrm{p}=0,047\end{array}$ & $\mathrm{p}=0,000$ \\
\hline
\end{tabular}

* - differences are significant compared with the control $(p<0.05)$

** - differences are significant compared with group $1(p<0.05)$

$\#-$ differences in the level of statistical trends $(0.1>p>0.05)$

same time, in the control group there was also an inverse relationship between RANKL and OPG/RANKL ( $\mathrm{r}=-0.58$, $\mathrm{p}=0.00017)$.

A moderate negative correlation between TGF- $\beta 1$ and OPG levels was found in the control group $(\mathrm{r}=-0.36, \mathrm{p}=$ 0.027 ), in group 1 there was a strong positive relationship between TGF- $\beta 1$ and the OPG/RANKL ratio $(r=0.66$, $\mathrm{p}=0.000$ ) (Fig. 2). In the control group, a moderate strength negative correlation was found between IL- 6 and OPG levels $(\mathrm{r}=-0.62, \mathrm{p}=0.000)$. In group 1 , positive correlations of the average force between IL- 6 and OPG $(r=0.67, p=$ $0.000)$, IL- 6 and RANKL $(r=0.53, p=0.001)$, IL- 6 and OPG/RANKL $(r=0.39, p=0.016)$. At the same time, there were no significant correlations between the similar indicators in patients of the 2 groups.

\section{DISCUSSION}

There is evidence that serum OPG levels increase with age, as well as in patients with osteoporosis. The age-related increase in OPG as an antiresorptive factor may reflect a failure of the paracrine mechanism for compensating for bone loss. In osteoporosis, an increase in the level of OPG is a compensatory response to enhanced osteoclastic bone resorption [8].

In our study, a decrease in the level of OPG in patients of group 2 was found. A decrease in the level of the main blocker of osteoclast activity may indicate a breakdown of compensatory mechanisms due to a decrease in adaptive reserves. However, it should be borne in mind that our study was conducted 3 weeks after the injury, i.e. during the healing of a fracture. Therefore, a decrease in serum OPG may reflect the corresponding remodeling step, since the regeneration of bone tissue after a fracture is a complex cascade process, not limited to the synthesis process.

The level of OPG in patients of the 1st group was not statistically significantly different from its level in the control group. But in the 2nd group, where the treatment results were worse, it was significantly lower. It can be assumed that a low level of OPG in these conditions of bone turnover may indicate an increase in the risk of complications in the long term. OPG, first described as an inhibitor of osteoclastic bone resorption, performs many functions, including an anti-inflammatory effect [9], and stimulates angiogenesis [10]. Taking into account the anti-inflammatory effect of OPG, a decrease in its concentration in serum may contribute to the insufficiency of anti-inflammatory regulatory factors, which may increase the risk of an adverse outcome.

It is believed that the nature of bone remodeling is largely determined by the ratio of OPG and RANKL products. The decrease in the OPG/RANKL ratio in patients of groups 1 and 2 indicates the preservation of increased resorptive activity characteristic of the initial stages of osteoreparation. However, at this stage, activation of synthetic processes is of key importance, since the result will be determined by the optimal ratio of synthesis and resorption. Delay and insufficient level of activity of synthetic processes can be an unfavorable prognostic sign for stable long-term fixation of the components of the endoprosthesis. It is the balance of bone formation and resorption in remodeling cycles that determines the optimal ratio of reparative and resorptive processes at the endoprosthesis - bone interface.

TGF- $\beta 1$ is one of the main growth factors involved in the multi-stage healing process of bone fractures at various stages of ossification. TGF- $\beta 1$ promotes matrix formation and differentiation of osteoblasts, reduces the ability of osteoblasts to secrete the differentiation factor of osteoclasts RANKL, thereby indirectly restricting the further formation of osteoclasts and can affect bone mass [11]. TGF- $\beta 1$ contributes to the activation of myofibroblasts [12], which play a key role in wound healing. The highest concentration of TGF- $\beta 1$ was registered in patients of group 1 , whose long-term results of treatment were better, which confirms the assumption that a more adequate response to bone damage is formed in patients of this group aimed at activating the osteoreparation process.

After bone resorption occurs, TGF- $\beta$ is locally released 


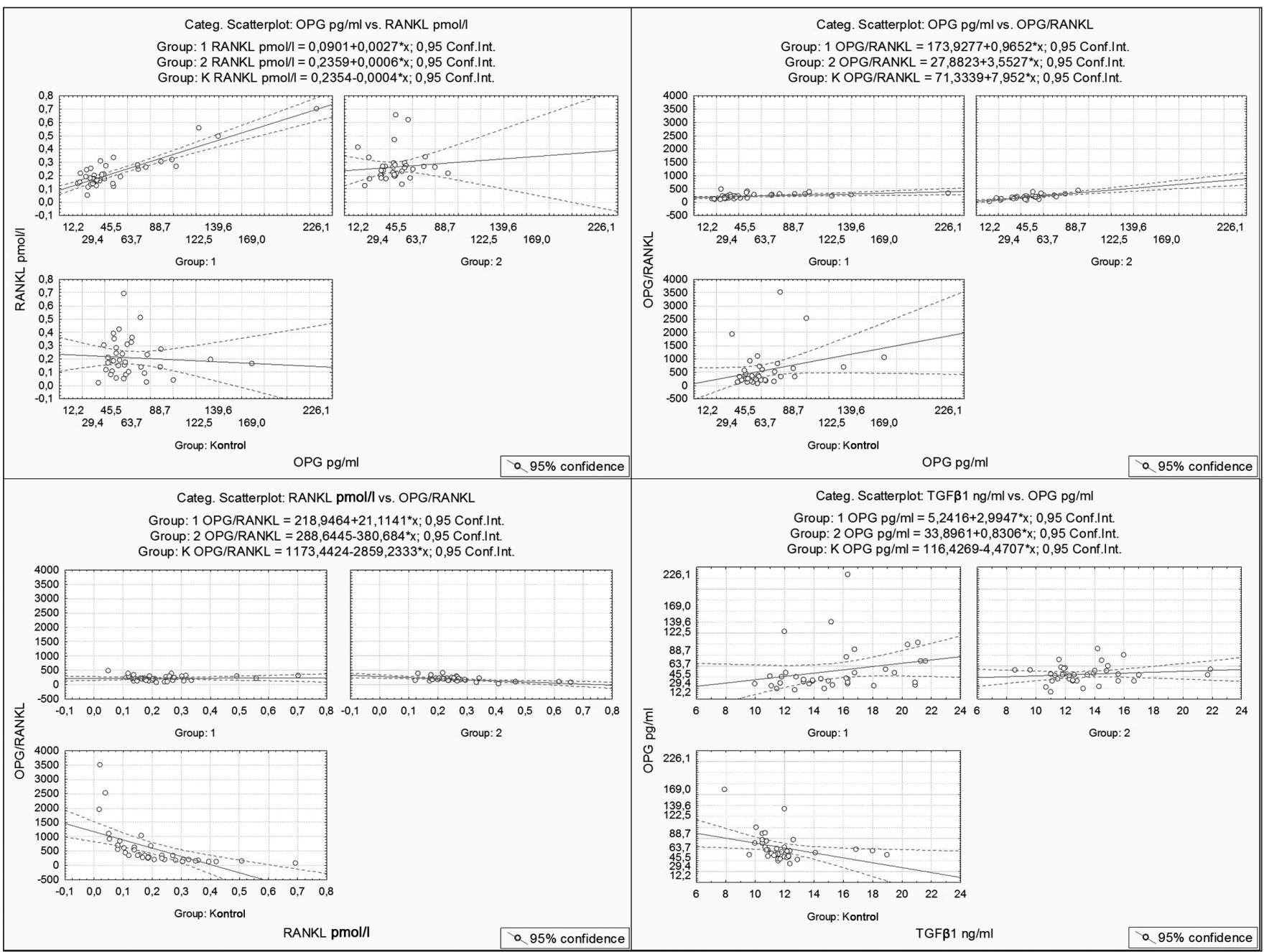

Figure 1. Correlations between $O P G$ and RANKL, OPG and OPG/RANKL, RANKL and OPG/RANKL, TGF- $\beta 1$ and OPG markers are presented.

from the bone matrix and then partially inhibits bone resorption, causing osteoclast apoptosis, and partly stimulating osteoblastic bone formation [13]. TGF- $\beta$ released during osteoclastic resorption (osteoplastic resorption) can then induce the expression of OPG osteoblasts in the local bone microenvironment and thereby inhibit the formation / activity of osteoclasts and stop bone resorption [14]. It can be assumed that a higher level of TGF- $\beta 1$ in patients of group 1 allows you to maintain a more active expression of OPG and indirectly - a higher bone turnover. These data suggest that in patients with a more favorable prognosis, a shift in the synthesis / resorption balance occurs both by increasing the synthetic activity and by reducing the level of resorption, which are regulated by TGF- $\beta 1$ and OPG, respectively.

There is evidence that the level of TGF- $\beta 1$ increases with various inflammatory reactions, fibrous changes in the liver, kidneys, myocardium [15]. This should be taken into account when evaluating the results, since our study included patients of old and old age with the presence of concomitant diseases. In a study by Hara Y. et al. (2017) showed that in the group of patients with normal fracture of the fracture, the maximum peak of TGF- $\beta 1$ was ob- served 2 weeks after the injury, and in the group without adhesion, a delayed maximum peak was observed after 3 weeks [5]. It is also possible that the very presence of an implant leads to a local release of TGF- $\beta$ by osteoblasts. [6] . Therefore, further studies are required on the expression of TGF- $\beta 1$ in the dynamics of the regeneration process of the damaged bone, taking into account the chronological sequence of its stages.

An increase in the level of IL-6 in patients of both groups relative to the control was an expected and regular result, since IL-6 is produced as an acute phase protein to stimulate the immune response. IL-6 is considered a key mediator in this complex scenario due to its significant correlation with injury severity and clinical complications [16].

However, the duration and relative variability of the morbidity of the intervention cannot be considered as a determining factor in the dynamics of IL-6 increase. The most important factor regulating the inflammatory response to surgery is the patient's age [17]. One of the manifestations of the age-related problem of decreasing physiological reserves is a decrease in adrenocortical sensitivity to surgical stress, which is characteristic of the elderly against the background of an increase in the baseline 


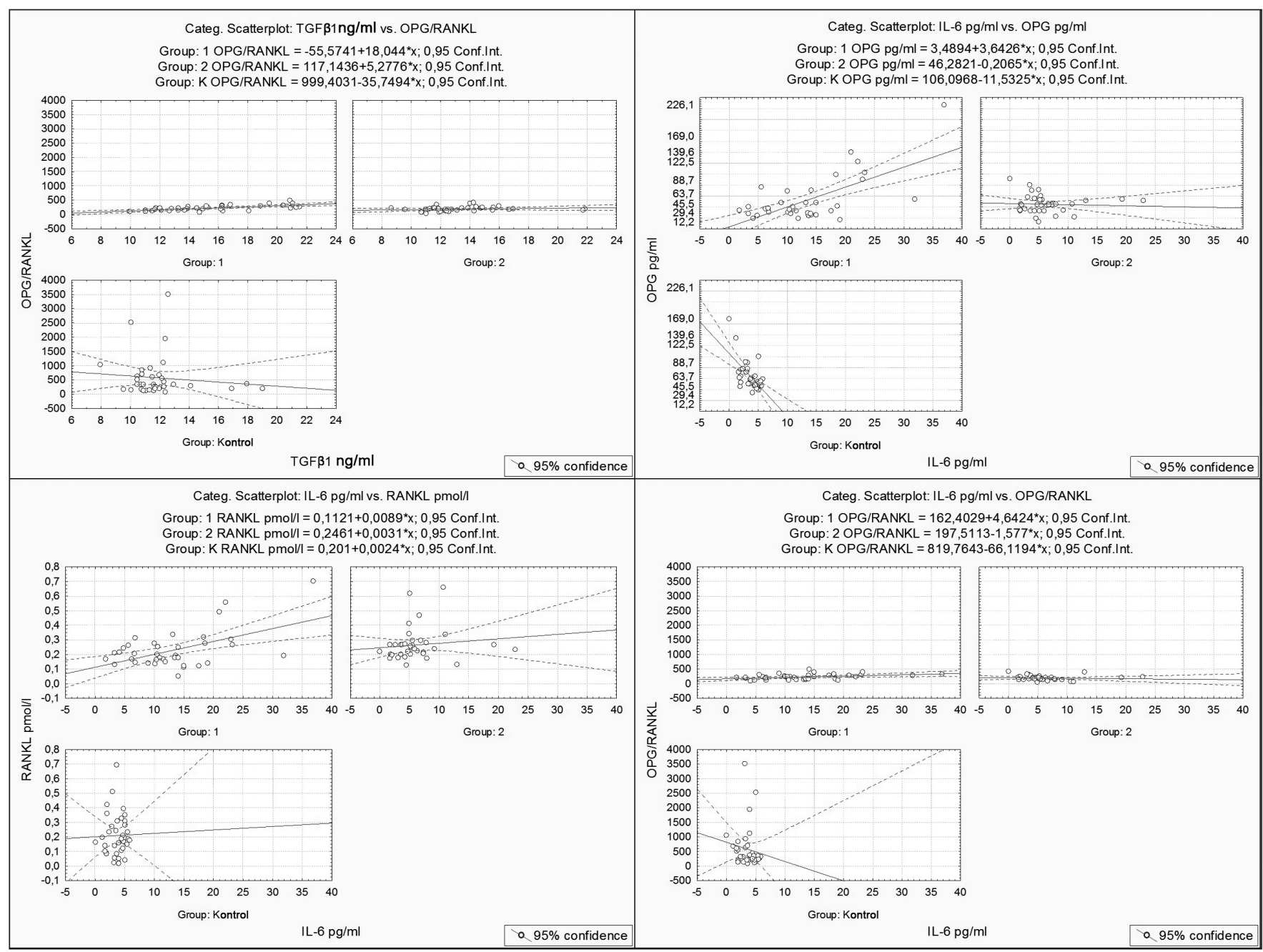

Figure 2. Correlations between TGF- $\beta 1$ and OPG/RANKL, IL- 6 and OPG, IL- 6 and RANKL, IL- 6 and OPG/RANKL.

level of cortisol [18]. This increase may lead to a decrease in the synthesis of OPG, increased expression of RANKL and inhibition of the synthesis of IL-6 [19]. These data are consistent with the observed decrease in the level of serum OPG and lower levels of IL-6 in patients of group 2 relative to group 1. Cytokine expression distorted in this way leads to impaired bone remodeling, leading to an increase in the rate of resorption without compensatory bone formation, and as a result, a rapid loss of bone matter.

Expression of IL-6 in fracture conditions is considered to be biphasic. It peaks during the acute inflammatory phase after a fracture, when it causes the recruitment of immune cells and stimulates angiogenesis. Later, IL- 6 expression increases again due to remodeling of the bone during its secondary formation [20]. The relatively low level of serum IL- 6 in patients of group 2 can be explained by the insufficient response of immune and regulatory mechanisms to damage. In addition, this may be due to a violation of the process of stress adaptive remodeling in the form of a delay in the stage at which it can regulate intramembranous and endochondral ossification [20]. There is experimental evidence that inhibition of IL-6 at an early stage after a fracture reduces systemic inflammation, recruitment of immune cells and bone regeneration, which leads to delayed healing of the fracture [21].

In addition, IL-6 is a pleiotropic cytokine that has not only pro-inflammatory but also anti-inflammatory effect. IL-6 initiates a fracture healing cascade and can also play a key role in the remodeling phase [22]. Therefore, a relatively low level of its content in the blood serum of patients of group 2 may be a manifestation of the changing nature of the adaptive response due to the violation of the regulatory role of this cytokine in the complex cascade of fracture healing.

The expression ratio of OPG and RANKL is critical in regulating osteoclast activity during bone remodeling. In our study, the relationship between OPG and RANKL levels was found only in group 1 . In group 2 (treatment outcome is worse) and in the control this relationship was absent (Fig. 1). Consequently, the differences in the change of remodeling processes between these groups are minimal, which can be considered as the lack of an adequate response to damage in patients of group 2. It can be assumed that the activation of osteoclasts along the regulatory path RANK/RANKL/OPG during bone remodeling in patients of group 1 is more coordinated than in other groups. Since 
the control group did not undergo surgery, similar indicators of the regulatory pathway in patients of group 2 may indicate insufficient activation of the remodeling process and, accordingly, the processes of osteointegration and implant engraftment.

In the control group, there is no correlation between the OPG level and the OPG/RANKL ratio; there is a negative correlation between RANKL and OPG/RANKL (Fig. 1). In patients of group 1 with a more favorable long-term prognosis, an inversion of these relationships is observed: there is a correlation between OPG and OPG / RANKL, and between RANKL and OPG/RANKL there is no. Thus, the resorptive activity, characterized by the OPG/RANKL ratio, in the control and in patients of group 1 is realized through different regulatory pathways. In group 2, where the results of treatment were worse, the effect of both RANKL and OPG on resorptive activity was observed to about the same extent.

It is worth considering the fact that TGF- $\beta$ regulates osteoclastogenesis and osteoclast survival, in particular, through the induction of OPG, which is known to inhibit their formation and functioning [14.]. The lack of correlation between the levels of TGF- $\beta 1$ and OPG in patients of both groups, characteristic of the control group (Fig. 2), indicates insufficient activation of reparative processes. Under physiological conditions, a balance is achieved due to the fact that changes in the production of TGF- $\beta 1$ and OPG balance each other. The presence of a negative correlation between these indicators in the control group can probably be explained by the fact that the activation of synthetic processes in bone due to an increase in the level of TGF- $\beta 1$ results in a compensatory decrease in the level of OPG, which simultaneously leads to the activation of osteolytic processes, which maintains bone homeostasis. In terms of injury, this compensatory mechanism is no longer valid. In the case of injury, the relationship between these two regulatory paths is broken, which results in the absence of a correlation between these two indicators. This may partly explain the correlation between TGF- $\beta 1$ and OPG in the control group and the absence of such after injury.

The presence of a correlation between TGF- $\beta 1$ and OPG/ RANKL in patients of group 1 suggests that the processes of resorption and restoration of damaged bone are more balanced. In this case, we can talk not only about the activation of osteoblastic bone formation processes in which TGF- $\beta 1$ is involved, but also about the synchronous activation of the regulatory path RANK/RANKL/OPG, which inhibits osteoclast activity and accordingly provides a shift in remodeling towards consolidation of the implant. The absence of significant correlations in the control and in group 2 can be interpreted as insufficient activation of bone remodeling processes in conditions of injury, which can lead to longer healing periods.

The negative correlation between IL- 6 and OPG in the control group may be related to the fact that an increase in IL-6 levels due to changes in intercellular signaling in aging conditions and the development of chronic inflammatory background is a factor that provokes the activation of the resorptive process in bone, which is realized through a decrease in OPG level. As a result of this kind of relationship through other regulatory paths, a compensatory increase in the level of OPG may occur, which is characteristic of elderly and senile age [8].

In group 1 , there is a change in the direction of the relationship between IL-6 and OPG compared to control group, the correlation becomes positive. In this group, there is also a correlation between IL- 6 and RANKL. It can be assumed that at this stage both the pro-inflammatory effect of IL- 6 and its participation in adaptive remodeling is manifested. However, the positive correlation between IL- 6 and the OPG/RANKL ratio suggests that IL- 6 activates both resorptive and synthetic processes, but at this stage its influence on the activation of osteosynthesis prevails. Patients 2 groups of such patterns were not observed, which may indicate a violation of the coordination of reparative processes.

\section{CONCLUSIONS}

The data obtained confirm the well-known position that after endoprosthesis replacement of large joints, both resorption and bone formation is activated. In patients with a less favorable outcome, the initial metabolic abnormalities in the bone tissue are significantly aggravated, consisting in the relative predominance of the process of bone resorption over bone formation. These changes are generally characterized by the imbalance in the activation of signaling pathways due to the disruption of the synthesis of regulatory molecules.

A key factor in the favorable outcome of treatment is the coordination of activation of regulatory pathways. Therefore, in our opinion, the prediction of results should be primarily based on an assessment of these relationships, taking into account changes in individual parameters. The results of treatment are largely determined by the violation of the regulatory mechanisms of osteoinductive activity of bone tissue.

\section{REFERENCES}

1. Kalchenko AV, Babalyan VA, Khvysyuk AN, Gurbanova TS, Cherepov VV. Analysis of surgical treatment in elderly and senile patients with proximal femur fractures by extracortical plate osteosynthesis. Trauma. 2017;18(3):80-85. (in Russian).

2. Pavlov SB, Pavlova GB, Veligotsky AN, Zukow W. Features cytokine profile in patients with pathological processes in obstuktivnymi pancreatoduodenal area in terms of reactions of functional system for damage connective tissue. Journal of Education, Health and Sport. 2016;6(7):610-629. (in Russian).

3. Pavlov SB, Kumechko MV, Litvinova OB, Babenko NM, Goncharova AV. Bone regulatory mechanisms destruction in experimental chronic kidney disease. Fiziol Zh. 2016;62(3):54-59. (in Ukrainian).

4. Coondoo A. The role of cytokines in the pathomechanism of cutaneous disorders. Indian J Dermatol. 2012;57(2): 90-96.

5. Hara Y, Ghazizadeh M, Shimizu H, et al. Delayed expression of circulating TGF- $\beta 1$ and BMP-2 levels in human nonunion long bone fracture healing. J Nippon Med Sch. 2017;84(1):12-18. 
6. Pacheco-Martelo V, Roldán-Vasco S. Enzymes and cytokines disease in total hip arthroplasty: promoters of immune loosening. Rev Fac Med. 2018;66(3):477-484.

7. Harris WH. Traumatic arthritis of the hip after dislocation and acetabular fractures: treatment of mold arthroplasty. An end-result study using a new method of result evaluation. J Bone Joint Surg Am. 1969; 51(4):737-755.

8. Pandey A, Khan YA, Kushwaha SS, Mohammed F, Verma A. Role of serum osteoprotegerin as a diagnostic indicator of primary osteoporosis in perimenopausal and postmenopausal women: An Indian perspective. Malays Orthop J. 2018;12(1):31-35.

9. Escobar-Morreale HF, Botella-Carretero Jl, Martinez-García MA, LuqueRamírez M, Alvarez-Blasco F, San Millán JL. Serum osteoprotegerin concentrations are decreased in women with the polycystic ovary syndrome. Eur J Endocrinol. 2008;159(3):225-232.

10. Rochette L, Meloux A, Rigal E, Zeller M, Cottin Y, Vergely C. The role of osteoprotegerin and its ligands in vascular function. Int J Mol. Sci. 2019;20(3):705.

11. Chen G, Deng C, Li YP. TGF- $\beta$ and BMP signaling in osteoblast differentiation and bone formation. Int J Biol Sci. 2012;8(2), 272-288.

12. Rosin NL, Falkenham A, Sopel MJ, Lee TD, Légaré JF. Regulation and role of connective tissue growth factor in Angll-induced myocardial fibrosis. Am J Pathol. 2013;182(3):714-726.

13. Houde N, Chamoux E, Bisson M, Roux S. Transforming growth factorbeta1 (TGF-beta1) induces human osteoclast apoptosis by up-regulating Bim. J Biol Chem. 2009;284(35):23397-23404.

14. Thirunavukkarasu K, Miles RR, Halladay DL, et al. Stimulation of osteoprotegerin (OPG) gene expression by transforming growth factorbeta (TGF-beta). Mapping of the OPG promoter region that mediates TGF-beta effects. J Biol Chem. 2001;276(39):36241-36250.

15. Kostiv SG, Kalinichenko NYu, Matveeva RE. Trophic factors of bone growth, their morphogenetic characterization and clinical significance. Pacific Medical Journal. 2017;1:10-16. (in Russian).

16. Alper B, Erdogan B, Erdogan M0, Bozan K, Can M. Associations of trauma severity with mean platelet volume and levels of systemic inflammatory markers (IL1 $\beta$, IL6, TNFa, and CRP). Mediat Inflamm. 2016;ID9894716:1-4.

17. Minetto MA, Oprandi G, Saba L, et al. Serum interleukin-6 response to elective total hip replacement surgery. Int Orthop. 2006;30(3):172-176.

18. Beale E, Zhu J, Belzberg H. Changes in serum cortisol with age in critically ill patients. Gerontology. 2002;48:84-92.

19. Pavlov SB, Babenko NM, Kumetchko MV, Litvinova OB. Violations of cell-molecular mechanisms of bone remodeling under influence of glucocorticoids. Regulatory Mechanisms in Biosystems. 2018;9(1):124-129.
20. Ai-Aql ZS, Alagl AS, Graves DT, Gerstenfeld LC, Einhorn TA. Molecular mechanisms controlling bone formation during fracture healing and distraction osteogenesis. J Dent Res. 2008;87(2):107-118.

21. Prystaz K, Kaiser K, Kovtun A, et al. Distinct effects of IL-6 classic and trans-signaling in bone fracture healing. Am J Pathol. 2018;188(2):474-490.

22. Kon TJ, Cho T, Aizawa M, et al. Expression of osteoprotegerin, receptor activator of NF-kappaB ligand (osteoprotegerin ligand) and related proinflammatory cytokines during fracture healing. J Bone Miner Res. 2001;16:1004-1014.

\section{Funding}

This study was funded by the Ministry of Health of Ukraine for the state budget.

\section{ORCID and contributionship:}

Oleksandr M. Khvysyuk - 0000-0002-4826-6567 A,D,E,F

Volodymyr O. Babalian - 0000-0003-4149-2542 A,B,E,F

Serge B. Pavlov - 0000-0002-3952-1511 A,C,E,F

Galina B. Pavlova - 0000-0002-3941-2171 A,B,D,E

\section{Conflicts of interest:}

Authors declare no conflict of interest.

\section{CORRESPONDING AUTHOR \\ Serge B. Pavlov \\ Kharkiv Medical Academy of Postgraduate Education \\ 58 Amosova street, 61176, Kharkiv, Ukraine \\ tel: +380577118045 \\ e-mail:psb@ua.fm}

Received: 08.04.2019

Accepted: 29.11.2019

A - Work concept and design, B - Data collection and analysis, C - Responsibility for statistical analysis, D-Writing the article, $\mathbf{E}-$ Critical review, $\mathbf{F}$ - Final approval of the article 